\title{
Evaluation of the Diagnostic Value of the Ultrasound ADNEX Model for Benign and Malignant Ovarian Tumors
}

\author{
Xiao-Shan Peng* \\ Yue $\mathrm{Ma}^{*}$ \\ Ling-Ling Wang \\ $\mathrm{Hai}-\mathrm{Xia} \mathrm{Li}$ \\ Xiu-Lan Zheng \\ Ying Liu
}

Department of Ultrasound, Harbin Medical University Cancer Hospital, Harbin, 150080, People's Republic of China

*These authors contributed equally to this work
Correspondence: Ying Liu

Department of Ultrasound, Harbin Medical University Cancer Hospital,

No. 150 of Haping Road, Nangang

District, Harbin, I50080, People's

Republic of China

$\mathrm{Tel} / \mathrm{Fax}+86045185718392$

Email liuy_dr@I63.com
Objective: To investigate the diagnostic performance of the ADNEX model in the International Ovarian Tumor Analysis diagnostic models for ovarian tumors and further explore its application value in the staging of ovarian tumors.

Methods: A total of 224 patients who underwent ultrasound for evaluation of adnexal masses and were treated surgically owing to adnexal masses from January 2018 to June 2020 in our hospital were selected for research on the diagnostic accuracy of the ADNEX model. The clinical information and ultrasonographic findings of the patients were collected, and the pathological diagnosis was taken as the gold standard. According to the ADNEX model, the ovarian tumors were divided into five subtypes: benign and borderline, stage I, stage II-IV, and metastatic cancer. The sensitivity, specificity, positive predictive value, negative predictive value, diagnostic odds ratio, and area under the receiver operating characteristics curve (AUC) of the ADNEX model were calculated.

Results: Of the 224 patients, 119 (53.1\%) developed benign tumors and 105 (46.9\%) had malignant tumors. When the cut-off value for malignancy risk was $10 \%$, the ADNEX model including CA 125 achieved a sensitivity of 94.3\% (95\% CI: 88.0-97.9\%), specificity of $74.0 \%$ (95\% CI: $65.1-81.6 \%$ ), positive predictive value of $76.2 \%$ (95\% CI: $70.2-81.3 \%$ ), negative predictive value of $93.6 \%$ (95\% CI: 87.0-97.0\%), diagnostic odds ratio of 45.25 , and an AUC of 0.94 (95\% CI: 0.90-0.97) for differentiating between benign and malignant ovarian tumors. The AUC in the model excluding CA 125 was 0.93 (95\% CI: $0.89-0.96$ ), but the difference was not statistically significant $(\mathrm{P}=0.20)$. The accuracy of the ADNEX model for the diagnosis of ovarian tumors of all subtypes exceeds $80 \%$ when CA 125 measurements were included in the application, but the sensitivity for diagnosing borderline, stage $\mathrm{I}$, and metastatic ovarian tumors was only $60.0 \%$ (95\% CI:36.1-80.9\%), 28.6\% (95\% CI:8.4$58.1 \%)$ and $45.5 \%$ (95\% CI:16.7-76.6\%).

Conclusion: The ADNEX model shows good diagnostic performance in differentiating between benign and malignant ovarian tumors. The model has a certain clinical value in the diagnosis of all subtypes of ovarian tumors, but the sensitivity is unsatisfactory for the diagnosis of borderline, stage I, and metastatic ovarian tumors and needs to be verified.

Keywords: IOTA, ADNEX model, ovarian tumors, ultrasound

\section{Introduction}

The ovarian tumor is a common gynecological disease that has diverse pathological types. Relevant papers have reported that malignant ovarian tumors contribute the highest mortality rate among malignant gynecological tumors, with a poor prognosis. ${ }^{1-4}$ 
Many patients with small early stage tumors are asymptomatic, and most patients do not consult a doctor until they are in the middle and advanced stages and develop symptoms, such as abdominal pain, abdominal distension, and menstrual disorders, resulting in a serious threat to the lives and health of women. However, studies have shown that patients with stage I ovarian cancer have a five-year survival rate of up to $90 \%$ after treatment. ${ }^{1}$ Therefore, early detection and treatment are crucial to improving the survival rate of patients with ovarian cancer. $^{5}$

Ultrasonography plays an important role in the imaging examination of ovarian tumors. Ultrasonography does not incur any radiation exposure and is relatively inexpensive compared to other imaging modalities. Therefore, it is often used as the preferred imaging method for gynecological diseases. The International Ovarian Tumor Analysis (IOTA) Group proposed multiple models and rules to describe benign and malignant ovarian tumors by using the ultrasonographic features of ovarian tumors. The ADNEX model is one of the latest developments of the IOTA Group and it is a multi-classification model to evaluate ovarian tumors. It proposes standardized terms to describe the characteristics of ovarian tumors and aims to predict whether a tumor is benign or malignant but also for staging malignant masses. ${ }^{6,7}$ The ADNEX model uses three clinical predictors and six ultrasound predictors and classifies tumors into benign, borderline, stage I primary invasive cancer, stage II-IV primary invasive cancer, and secondary metastatic cancer. The evaluation results are presented as a percentage of each subtype and a relative risk value.

The diagnostic value and application prospects for the ADNEX model for ovarian tumors currently deserves exploration. The purpose of this study is to evaluate the diagnostic performance of the ADNEX model for differentiating benign from malignant ovarian tumors, both overall and specifically for borderline, stage I, and metastatic ovarian tumors.

\section{Materials and Methods}

A total of 270 patients who underwent ultrasound for evaluation of adnexal masses and were treated surgically owing to adnexal masses examined for adnexal masses at the Harbin Medical University Cancer Hospital from January 2018 to June 2020 were analyzed retrospectively. This Hospital is a regional tertiary oncology diagnostic center. Of them, 46 patients were excluded for the following reasons: no indication for surgery, pregnancy, incomplete data, doubtful histology of biopsy sample, failure to undergo surgery within 120 days of the ultrasound examination, and 224 patients were selected as the study subjects. The inclusion criteria were as follows: at least one adnexal mass, received ultrasonographic and CA 125 examinations and surgical treatment at our hospital, and obtained postoperative pathological diagnostic results. If adnexal masses were found bilaterally, one mass with the most complex morphology was analyzed. If the masses have a similar morphology, the mass with the greatest diameter was selected for determining malignancy. The main symptoms at the time of consultation included abdominal mass, distension, and pain and menstrual disorders.

The color Doppler diasonograph (Samsung WS80A) was used, with an abdominal probe frequency of 3-7 $\mathrm{MHz}$ and intracavitary probe frequency of 5-9 MHz. All ultrasound examinations are completed by the same team who were Level III proficient in gynecologic sonography with more than 15 years of experience in gynecological ultrasound diagnosis assessed the ultrasonographic characteristics of tumors based on the nomenclature and methodology proposed by the IOTA Group. ${ }^{8}$ The first choice of examination method is transvaginal ultrasound examination. It was advised that patients with a large lesion or those without a sexual history should have a well-filled bladder in order to receive an abdominal examination. The ADNEX model is available as part of the built-in mobile software of the diasonograph, or online (http://www.iotagroup.org/adnexmodel). The ADNEX model consists of three clinical predictors and six ultrasound predictors. The clinical predictors include age (years), oncology center (yes/no), and serum CA 125 measurement results. The ultrasound predictors include the maximal diameter of the tumor ( $\mathrm{mm})$, the maximal diameter of the solid tissue $(\mathrm{mm})$, whether the tumor contains more than 10 cyst locules (yes/no), the number of papillary projections on the cyst wall $(0,1,2,3$, or $>3)$, the presence of acoustic shadows (yes/no), and the presence of ascites (yes/no). Collected the related clinical information (age, serum CA125-II levels, oncology center yes/no) of the patients, successively inputted clinical and ultrasonographic predictors of the patients into the system, and clicked the calculation button to allow the software to automatically analyze the nature and stage of the tumor. The results were presented as the maximum risk of malignancy value and the relative risk value of each subtype. The overall risk predictive value of a malignant tumor is the sum of the risk values of all the subtypes of malignancies including borderline, stage I, stage II-IV, and metastatic cancer. The original study showed that the model could be used conduct malignancy risk evaluation for tumors 
even in the absence of CA 125 measurements. ${ }^{6}$ The ultrasonographer was blinded to the histological diagnosis.

Case: A 55-year-old female patient presented with a mixed mass in the adnexal area. The hospital attended is the Oncology Centre.The mass had a maximal diameter of $136 \mathrm{~mm}$, and the solid tissue had a maximal diameter of $37 \mathrm{~mm}$. There were less than 10 cyst locules in the lesion and over 3 papillary projections in the cyst locules. No acoustic shadows were present posteriorly, and no ascites was present. The CA 125 value was $367 \mathrm{U} / \mathrm{mL}$. Based on the calculations of the ADNEX model, the overall malignancy risk value for the patient was $92.4 \%$, and the relative risk index of borderline cancer was the highest $(\mathrm{R} R=3.66)$. (see Figure 1) (Informed consent has been obtained from the patient).

Serum CA125 was measured by Elecsys CA125 II (Roche Diagnostics GmbH, Germany) immunoassay kit through the electrochemiluminescence technic according to the manufacturer's instructions. A cut-off value of $35 \mathrm{U} / \mathrm{mL}$ was used.

All patients enrolled in this study were treated surgically at our hospital and received final pathological results.
The postoperative pathological staging was subject to the International Federation of Gynecology and Obstetrics. ${ }^{9}$

The statistical software packages SPSS 26.0 and MedCalc 19.5.6 were used for data analysis. For statistical purposes, borderline tumors were considered as malignant. The quantitative data were expressed as medians (interquartile range) and the categorical data were expressed as frequencies and percentages. Ultrasound characteristics and clinical data were compared between benign and malignancy by the chi-square tests for categorical variables, and the Mann-Whitney $U$-test for continuous variables. The overall malignancy risk predictive value was used to calculate the area under the receiver operating characteristics curve (AUC). The diagnostic performance of the ADNEX model for benign and malignant ovarian tumors with and without CA 125 was analyzed by calculating the sensitivity, specificity, positive predictive value, negative predictive value, and diagnostic odds ratio, with a 95\% confidence interval. The differences between the two situations were analyzed using the DeLong's test, and $\mathrm{P}<0.05$ was

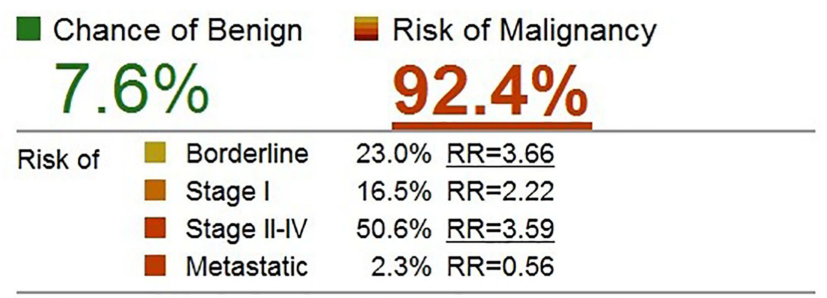

Risk of malignancy is $>=10 \%$ (elevated)

This feature is validated by International Ovarian Tumor Analysis (IOTA) group. This feature is a risk calculation providing statistical information (no diagnosis).

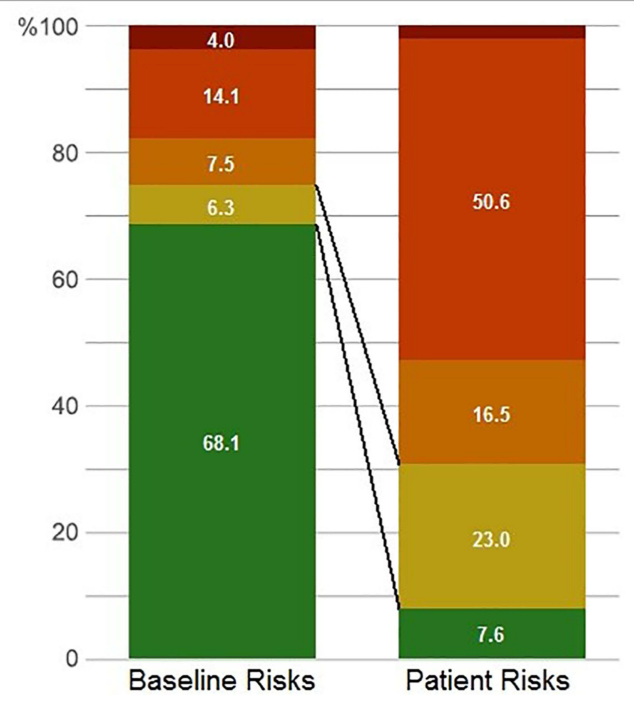

Age of the Patient

Oncology Center?

Max. Diameter of Lesion

Max. Diameter of Solid Part

More than 10 Locules?

Number of Papillations

Acoustic Shadows Present?

Ascites Present?

Serum CA-125
55

Yes

$136 \mathrm{~mm}$

$37 \mathrm{~mm}$

No

$4+$

No

No

$367 \mathrm{U} / \mathrm{m}$

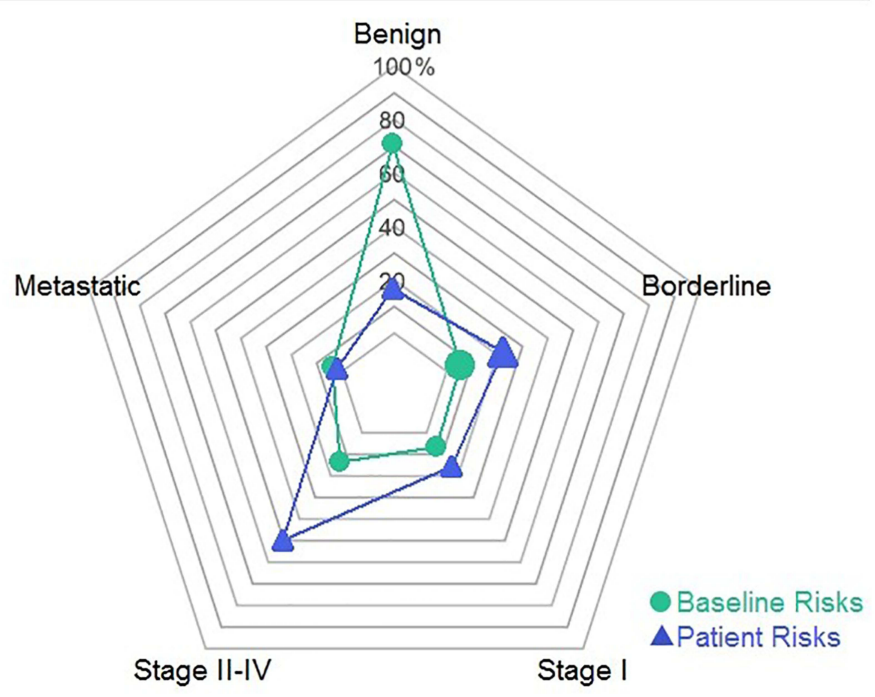

Figure I ADNEX calculation results. 
considered to be statistically significant. In addition, the diagnostic performance of the ADNEX model using different cut-off values for benign and malignant ovarian tumors was analyzed by calculating the sensitivity, specificity, positive predictive value, negative predictive value, and diagnostic odds ratio. Finally, the diagnostic capacity of the ADNEX model for malignant ovarian tumors and all subtypes was analyzed using a cut-off value for malignancy risk of $10 \% . P<0.05$ indicated statistical significance.

\section{Results}

The clinical and ultrasound predictors of the 224 patients are shown in Table 1. Patients in the malignant group were older than those in the benign group and had a higher serum CA 125 level $(\mathrm{P}<0.001)$. The maximal diameter of the lesion, maximal diameter of the solid tissue, and the incidence of papillary projections were significantly higher in the malignant group than in the benign group $(\mathrm{P}<$ 0.001). The number of lesions with more than 10 cyst locules was higher in the malignant group than in the benign group $(P<0.01)$. The incidence of ascites was approximately ten times higher in the malignant group than in the benign group ( $\mathrm{P}<0.001)$. In this study, no malignant lesions presented acoustic shadows $(\mathrm{P}<0.01)$.

A total of 224 patients with ovarian tumors were enrolled in this study, including 119 (53.1\%) with benign tumors and $105(46.9 \%)$ with malignant tumors. The pathological classification was subject to the WHO histological classification for ovarian tumors, ${ }^{10}$ and the pathological results are shown in Table 2. The most common benign tumor was a serous cystadenoma, and the most common malignant tumor was a serous cystadenocarcinoma. The pathological staging of malignant tumors was as follows: 20 patients $(8.9 \%)$ with borderline cancer, 14 (6.3\%) with stage I cancer, 60 (26.8\%) with stage II-IV cancer, and $11(4.9 \%)$ with metastatic cancer.

The diagnostic performance of the ADNEX model is shown in Table 3 and Figure 2. When the CA 125 measurements were included, the AUC of the ADNEX model was 0.94 (95\% CI: 0.90-0.97). When CA 125 measurements were excluded, the AUC was 0.93 (95\% CI: 0.89 0.96). The difference between these was not statistically significant $(\mathrm{P}=0.20)$. Optimal cut-off values were obtained to discriminate ovarian malignancy using the ADNEX model with the Youden index method. When the CA 125

Table I Clinical Indicators and Ultrasonic Manifestations of Ovarian Tumor Patients Were Summarized According to Pathological Stages

\begin{tabular}{|c|c|c|c|c|c|c|c|}
\hline \multirow[t]{2}{*}{ Characteristic } & \multirow{2}{*}{$\begin{array}{c}\text { Benign Tumor } \\
(n=119)\end{array}$} & \multicolumn{5}{|c|}{ Malignant Tumor $(n=105)$} & \multirow[t]{2}{*}{ p-value } \\
\hline & & $\begin{array}{l}\text { Borderline } \\
\text { Tumor } \\
(n=20)\end{array}$ & $\begin{array}{l}\text { Stage I } \\
(n=14)\end{array}$ & $\begin{array}{l}\text { Stage II-IV } \\
(n=60)\end{array}$ & $\begin{array}{l}\text { Metastatic } \\
\text { Tumor } \\
(n=1 I)\end{array}$ & $\begin{array}{c}\text { Total } \\
(n=105)\end{array}$ & \\
\hline Age (years) & $44(32-53)$ & $50(4 I-6 I)$ & $52(49-57)$ & $54(49-61)$ & $55(50-65)$ & $53(49-61)$ & $<0.001^{\mathrm{a}}$ \\
\hline CAI $25(\mathrm{U} / \mathrm{mL})$ & $20(13-44)$ & $37(\mid 2-221)$ & $138(32-333)$ & $547(230-1536)$ & $107(21-264)$ & $264(46-726)$ & $<0.001^{\mathrm{a}}$ \\
\hline $\begin{array}{l}\text { Maximal diameter of the } \\
\text { lesion }(\mathrm{mm})\end{array}$ & $72(47-103)$ & $97(67-138)$ & $118(70-138)$ & $116(91-148)$ & $83(58-117)$ & $110(7 \mid-144)$ & $<0.001^{\mathrm{a}}$ \\
\hline $\begin{array}{l}\text { Maximal diameter of the } \\
\text { solid tissue }(\mathrm{mm})\end{array}$ & $0(0-13)$ & $25(|I-3|)$ & $33(18-55)$ & $86(60-109)$ & $58(4 I-90)$ & $60(32-93)$ & $<0.001^{\mathrm{a}}$ \\
\hline $\begin{array}{l}\text { Papillary projections } \\
\text { present }\end{array}$ & $\mathrm{II}(9.2)$ & II (55.0) & $4(28.6)$ & $25(41.7)$ & $0(0.0)$ & $40(38.1)$ & $<0.001^{\mathrm{b}}$ \\
\hline 0 & $108(90.8)$ & $9(45.0)$ & $10(71.4)$ & $35(58.3)$ & II (I00.0) & $65(61.9)$ & \\
\hline 1 & $3(2.5)$ & $6(30.0)$ & $0(0.0)$ & $14(23.3)$ & $0(0.0)$ & $20(19.0)$ & \\
\hline 2 & $5(4.2)$ & $2(10.0)$ & $\mathrm{I}(7.1)$ & $2(3.3)$ & $0(0.0)$ & $5(4.8)$ & \\
\hline 3 & $\mathrm{I}(0.8)$ & $I(5.0)$ & $\mathrm{I}(7.1)$ & $3(5.0)$ & $0(0.0)$ & $5(4.8)$ & \\
\hline$>3$ & $2(1.7)$ & $2(10.0)$ & $2(14.3)$ & $6(10.0)$ & $0(0.0)$ & $10(9.5)$ & \\
\hline$>10$ cyst locules & $3(2.5)$ & $3(15.0)$ & $3(21.4)$ & $5(8.3)$ & $I(9.1)$ & $12(11.4)$ & $<0.01^{\mathrm{b}}$ \\
\hline Acoustic shadows & $12(10.1)$ & $0(0.0)$ & $0(0.0)$ & $0(0.0)$ & $0(0.0)$ & $0(0.0)$ & $<0.01^{\mathrm{b}}$ \\
\hline Ascites & $5(4.2)$ & $3(15.0)$ & $4(28.6)$ & $34(56.7)$ & $6(54.5)$ & $47(44.8)$ & $<0.001^{\mathrm{b}}$ \\
\hline
\end{tabular}

Notes: The data is presented as a median (quartile interval) or $\mathrm{n}(\%)$. $\mathrm{p}$-values of benign and malignant groups were calculated by: ${ }^{\mathrm{a}}$ Mann-Whitney $\mathrm{U}$-test; ${ }^{\mathrm{b}} \mathrm{Chi}$-square test. 
Table 2 Histopathological Findings of 224 Patients with Ovarian Tumor

\begin{tabular}{|l|c|}
\hline Histological Type & n (\%) \\
\hline Benign tumor & $119(53.1)$ \\
Serous cystadenoma & $32(14.3)$ \\
Mature cystic teratoma & $25(11.2)$ \\
Simple cyst & $18(8.0)$ \\
Mucinous cystadenoma & $14(6.3)$ \\
Endometrioma & $14(6.3)$ \\
Theca cell tumor & $5(2.2)$ \\
Struma ovary & $2(0.9)$ \\
Other benign ovarian tumors & $9(4.0)$ \\
\hline Borderline tumor & $20(8.9)$ \\
Serous cystadenoma & $13(5.8)$ \\
Mucinous cystadenoma & $7(3.1)$ \\
\hline Primary malignant tumor & $74(33.0)$ \\
Serous cystadenoma & $59(26.3)$ \\
Clear-cell carcinoma & $6(2.7)$ \\
Mucinous adenocarcinoma & $3(1.3)$ \\
Endometrioid adenocarcinoma & $3(1.3)$ \\
Carcinosarcoma & $3(1.3)$ \\
\hline Metastatic tumor & $11(4.9)$ \\
\hline
\end{tabular}

measurements were included in the ADNEX model, the best cut-off value of the model was $46.7 \%$.

The diagnostic efficacy of the ADNEX model with CA 125 measurements was evaluated with $5 \%, 10 \%, 20 \%, 30 \%$, and $46.7 \%$ as the cut-off values of malignant risk, respectively. When $10 \%$ was taken as the cut-off value for malignancy risk, the model had a sensitivity of $94.3 \%(95 \% \mathrm{CI}$ : $88.0-97.9 \%$ ), specificity of $74.0 \%$ (95\% CI: $65.1-81.6 \%$ ), positive predictive value of $76.2 \%$ (95\% CI: $70.2-81.3 \%$ ), negative predictive value of $93.6 \%$ (95\% CI: $87.0-97.0 \%$ ), and a diagnostic odds ratio of 45.25 . When $46.7 \%$ was taken as the cut-off value for malignancy risk, the model had a sensitivity of $86.7 \%$ (95\% CI: $78.6-92.5 \%$ ), specificity of

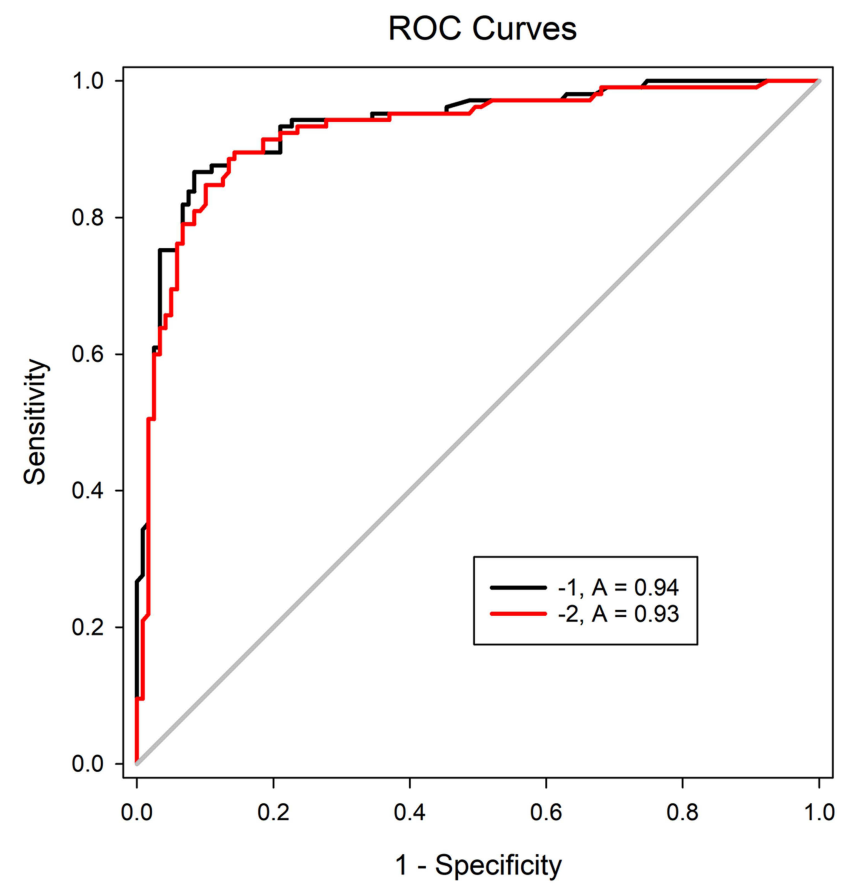

Figure 2 The diagnostic performance of the ADNEX model with (I) and without (2) CA 125.

91.6\% (95\% CI: $85.1-95.9 \%$ ), and a diagnostic odds ratio of 68.73. The evaluation results are shown in Table 4.

The diagnostic performance of the ADNEX model for all subtypes of ovarian tumors is shown in Tables 5 and 6 . The accuracy of the ADNEX model for the diagnosis of ovarian tumors of all subtypes exceeds $80 \%$, but the sensitivity for diagnosing borderline, stage I, and metastatic ovarian tumors was only $60.0 \%$ (95\% CI:36.1-80.9\%), $28.6 \% \quad(95 \% \quad \mathrm{CI}: 8.4-58.1 \%) \quad$ and $\quad 45.5 \% \quad(95 \%$ CI:16.7-76.6\%).

\section{Discussion}

Ultrasonography, especially vaginal ultrasonography, is currently the first choice for diagnosing gynecological

Table 3 Comparison of Diagnostic Performance of ADNEX Models with and Without CA I25

\begin{tabular}{|c|c|c|c|c|c|c|c|c|c|c|}
\hline $\begin{array}{l}\text { ADNEX } \\
\text { Models }\end{array}$ & $\begin{array}{c}\text { AUC } \\
(95 \% \mathrm{CI})\end{array}$ & $\begin{array}{c}\text { Sensitivity } \\
\text { (95\% CI) } \\
\text { (\%) }\end{array}$ & $\begin{array}{c}\text { Specificity } \\
\text { (95\% CI) } \\
\text { (\%) }\end{array}$ & $\begin{array}{c}\text { PPV } \\
(95 \% \mathrm{CI}) \\
(\%)\end{array}$ & $\begin{array}{c}\text { NPV } \\
(95 \% \mathrm{Cl}) \\
(\%)\end{array}$ & $\begin{array}{c}\text { LR+ } \\
(95 \% \mathrm{Cl})\end{array}$ & $\begin{array}{c}\text { LR- } \\
(95 \% \mathrm{CI})\end{array}$ & DOR & $\begin{array}{c}\text { Optimal } \\
\text { Cut-Off } \\
\text { Value (\%) }\end{array}$ & p-value* \\
\hline With CA 125 & $\begin{array}{c}0.94 \\
(0.90-0.97)\end{array}$ & $\begin{array}{c}86.7 \\
(78.6-92.5)\end{array}$ & $\begin{array}{c}91.6 \\
(85.1-95.9)\end{array}$ & $\begin{array}{c}90.1 \\
(83.3-94.3)\end{array}$ & $\begin{array}{c}88.6 \\
(82.7-92.7)\end{array}$ & $\begin{array}{c}|0.3| \\
(5.67-18.75)\end{array}$ & $\begin{array}{c}0.15 \\
(0.09-0.24)\end{array}$ & 68.73 & 46.7 & 0.20 \\
\hline Without CA 125 & $\begin{array}{c}0.93 \\
(0.89-0.96)\end{array}$ & $\begin{array}{c}89.5 \\
(82.0-94.7)\end{array}$ & $\begin{array}{c}85.7 \\
(78.1-91.5)\end{array}$ & $\begin{array}{c}84.7 \\
(78.0-89.6)\end{array}$ & $\begin{array}{c}90.3 \\
(84.1-94.2)\end{array}$ & $\begin{array}{c}6.27 \\
(4.02-9.78)\end{array}$ & $\begin{array}{c}0.12 \\
(0.07-0.22)\end{array}$ & 52.25 & 44.3 & \\
\hline
\end{tabular}

Notes: Diagnostic performance of International Ovarian Tumor Analysis ADNEX model in discriminating between benign and malignant tumors, according to whether CA 125 level is included in model. *DeLong's test was used to compare the AUC values of ADNEX model with and without CA I25 measurements.

Abbreviations: AUC, area under the receiver operating characteristic curve; PPV, positive predictive value; NPV, negative predictive value; LR+, positive likelihood ratio; LR-, negative likelihood ratio; DOR, diagnostic odds ratio. 
Table 4 Diagnostic Performance of the ADNEX Model with CA I25 at Different Cut-Off Values

\begin{tabular}{|l|c|c|c|c|c|c|c|}
\hline $\begin{array}{l}\text { Cut-Off } \\
\text { Value }\end{array}$ & $\begin{array}{c}\text { Sensitivity (95\% Cl) } \\
\mathbf{( \% )}\end{array}$ & $\begin{array}{c}\text { Specificity (95\% Cl) } \\
\mathbf{( \% )}\end{array}$ & $\begin{array}{c}\text { PPV (95\% Cl) } \\
\mathbf{( \% )}\end{array}$ & $\begin{array}{c}\text { NPV (95\% Cl) } \\
\mathbf{( \% )}\end{array}$ & LR+ (95\% CI) & LR- (95\% Cl) & DOR \\
\hline $5 \%$ & $95.2(89.2-98.4)$ & $60.5(51.1-69.3)$ & $68.0(62.9-72.7)$ & $93.5(85.8-97.2)$ & $2.41(1.92-3.02)$ & $0.08(0.03-0.19)$ & 30.13 \\
$10 \%$ & $94.3(88.0-97.9)$ & $74.0(65.1-81.6)$ & $76.2(70.2-81.3)$ & $93.6(87.0-97.0)$ & $3.62(2.66-4.92)$ & $0.08(0.04-0.17)$ & 45.25 \\
$20 \%$ & $92.4(85.5-96.7)$ & $79.0(70.6-85.9)$ & $79.5(73.2-84.7)$ & $92.2(85.7-95.8)$ & $4.40(3.09-6.26)$ & $0.10(0.05-0.19)$ & 44.00 \\
$30 \%$ & $89.5(82.0-94.7)$ & $80.7(72.4-87.3)$ & $80.3(73.8-85.6)$ & $89.7(83.2-93.9)$ & $4.63(3.19-6.73)$ & $0.13(0.07-0.23)$ & 35.62 \\
$46.7 \%$ & $86.7(78.6-92.5)$ & $91.6(85.1-95.9)$ & $90.1(83.3-94.3)$ & $88.6(82.7-92.7)$ & $10.31(5.67-18.75)$ & $0.15(0.09-0.24)$ & 68.73 \\
\hline
\end{tabular}

Notes: Diagnostic performance of International Ovarian Tumor Analysis ADNEX model with CA I 25 in discriminating between benign and malignant tumors at progressive cut-offs for overall risk of malignancy. Overal: Sum of risk for four subtypes of ovarian malignancy.

Abbreviations: PPV, positive predictive value; NPV, negative predictive value; LR+, positive likelihood ratio; LR-, negative likelihood ratio; DOR, diagnostic odds ratio.

Table 5 Comparison Between ADNEX Model Stage and Pathological Stage

\begin{tabular}{|l|c|c|c|c|c|}
\hline \multirow{2}{*}{ ADNEX Models } & \multicolumn{5}{|c|}{ Pathological Stage } \\
\cline { 2 - 6 } & Benign Tumor & Borderline Tumor & Stage I & Stage II-IV & Metastatic Tumor \\
\hline Benign tumor & 88 & 4 & 2 & 0 & 0 \\
Borderline tumor & 22 & 12 & 5 & 3 & I \\
Stage I & 3 & 1 & 4 & 5 & I \\
Stage II-IV & 6 & 2 & 3 & 4 & 4 \\
Metastatic tumor & 0 & I & 0 & \\
\hline
\end{tabular}

Table 6 Comparison of the Diagnostic Performance of the ADNEX Model for Different Subtypes of Ovarian Tumors

\begin{tabular}{|c|c|c|c|c|c|}
\hline ADNEX Models & $\begin{array}{c}\text { Sensitivity } \\
(95 \% \text { CI) (\%) }\end{array}$ & $\begin{array}{c}\text { Specificity } \\
(95 \% \mathrm{CI})(\%)\end{array}$ & $\begin{array}{c}\text { PPV } \\
(95 \% \mathrm{Cl})(\%)\end{array}$ & $\begin{array}{c}\text { NPV } \\
(95 \% \text { Cl) (\%) }\end{array}$ & $\begin{array}{c}\text { Diagnose } \\
\text { Accordance Rate } \\
(95 \% \mathrm{Cl})(\%)\end{array}$ \\
\hline Benign tumor & $74.0(65.1-81.6)$ & $94.3(88.0-97.9)$ & $93.6(87.0-97.0)$ & $76.2(70.2-81.3)$ & $83.5(78.0-88.1)$ \\
\hline Borderline tumor & $60.0(36.1-80.9)$ & $84.8(79.1-89.4)$ & $27.9(19.3-38.6)$ & $95.6(92.6-97.4)$ & $82.6(77.0-87.3)$ \\
\hline Stage I & $28.6(8.4-58.1)$ & $96.7(93.3-98.7)$ & $36.4(15.9-63.3)$ & $95.3(93.6-96.6)$ & $92.4(88.1-95.5)$ \\
\hline Stage II-IV & $85.0(73.4-92.9)$ & $90.9(85.4-94.8)$ & $77.3(67.5-84.8)$ & $94.3(90.0-96.8)$ & $89.3(84.5-93.0)$ \\
\hline Metastatic tumor & $45.5(16.7-76.6)$ & $97.7(94.6-99.2)$ & $50.0(25.3-74.7)$ & $97.2(95.3-98.3)$ & $95.1(91.4-97.5)$ \\
\hline
\end{tabular}

Abbreviations: PPV, positive predictive value; NPV, negative predictive value.

diseases,${ }^{11}$ and it can clearly show the internal structure of a lesion and its anatomical relationship with the peripheral tissues. However, the accuracy of diagnosis varies greatly based on the experience of the sonographer. ${ }^{12}$ The ADNEX model is the first-known multi-classification prediction model for evaluating ovarian tumors. ${ }^{13,14}$ Due to its ease of use, even inexperienced sonographers can give highly accurate diagnoses using the ADNEX model. ${ }^{5}$ The best way to diagnose the benignity or malignancy of an adnexal mass is by subjective assessment by an expert gynecologic ultrasonographer. ${ }^{3}$ Studies have shown that the diagnostic performance of the ADNEX model is consistent with the subjective evaluation of expert sonographers. ${ }^{15}$
Depending on the practice setting, preoperative determination of malignancy can be helpful in determining whether referral to a gynecologic oncologist is needed. Different subtypes of ovarian tumors are treated differently. BOT tumors confined to the ovary are associated with excellent survival, close to $100 \%$ in 10 years. Besides, they often affect young women, and relatively conservative treatments should be considered for patients who want to preserve their fertility. ${ }^{16-18}$ Metastatic ovarian tumors most commonly originate from the gastrointestinal tract or the breasts, and most patients have a low CA 125 level, and a few have papillary projections. Therefore, the determination of the primary lesion is of great importance in making treatment decisions ${ }^{19}$. In this study, the ADNEX model had good diagnostic ability for stage II-IV and 
benign ovarian tumors. Most especially in the diagnosis of stage II-IV ovarian tumors, the model had a high sensitivity $(85.0 \%)$ and specificity $(90.9 \%)$.

The ADNEX model had a better specificity in the diagnosis of borderline, stage I, and metastatic ovarian tumors $(84.8 \%$, $96.7 \%, 97.7 \%$ respectively), but its sensitivity was unsatisfactory. In particular, the sensitivity in the diagnosis of stage I ovarian tumors was only $28.6 \%$ (4/14 patients). The sensitivity of borderline and metastatic ovarian tumors is $60.0 \%(12 / 20$ patients) and $45.5 \%$ (5/11 patients) respectively, and these results are consistent with the results of Szubert et al. ${ }^{20}$ Therefore, the capacity of the ADNEX model for the diagnosis of borderline, stage I, and metastatic ovarian tumors needs to be improved. The false positive cases in the study mainly developed a serous or mucinous cystadenoma, and papillary projections may be present in these lesions. Mucinous cystadenoma often presents with the malignant feature of more than 10 cyst locules in the lesion. The false negative cases include four with borderline tumor and two with stage I cystadenocarcinoma and may be due to a lack of specific manifestations in the early stages of the lesions.

The diagnostic performance of the ADNEX model using different cut-off values was calculated in this study. With $10 \%$ as the malignant risk cut-off value, a good diagnostic odds ratio (45.25); a specificity of $74.0 \%$, which is slightly higher than the $71.3 \%$ obtained in the original study and the $67.7 \%$ in the multi-center external study; and a sensitivity of $94.3 \%$, which is lower than $96.5 \%$ obtained in the original study and similar to the $93.3 \%$ in the domestic external study ${ }^{5,6,17}$ were obtained. In this study, regardless of inclusion of CA 125 measurements, IOTA's ADNEX model exhibited a good ability to differentiate between benign and malignant ovarian tumors, with an AUC of 0.94 when CA 125 measurements were included and 0.93 when they were excluded $(\mathrm{P}=0.20)$. These results are supported by other study results. ${ }^{17}$ When CA 125 measurements were included in the ADNEX model, the best cut-off value of the model was $46.7 \%$. Taking this value as the cut-off value for malignancy risk, the model had a sensitivity of $86.7 \%$ (95\% CI: 78.6-92.5\%), specificity of 91.6\% (95\% CI: $85.1-95.9 \%$ ), and best diagnostic odds ratio of 68.73. In the study by Soo Jeong et al they identified the optimal cut-off point of discriminating ovarian malignancy using the ADNEX model at $90 \%$ sensitivity. The optimal cutoff point determined by the Youden index method in all participants was $47.3 \% .{ }^{15}$ In another non-tumor center, Tug et al study showed an optimal cut-off value of $14.05 \%$ exhibited more balanced results for sensitivity and specificity regardless of the patient's menopausal status. ${ }^{21}$
However, strict use of a fixed cutoff value may not be consistent with modern evidence-based medicine (EBM). ${ }^{7,22,23}$ Selecting cut-offs may be dependent on clinician, center, local protocols or guidelines. ${ }^{7}$ Greenhalgh et al indicated "real EBM demands individualized evidence, is characterized by expert judgment rather than mechanical rule following". ${ }^{23}$ Insisting on a fixed cutoff may not produce the best results and may even lead to unethical judgment. Some physicians may choose low cutoff values (eg 1-5\%) to maintain a high sensitivity and reduce cases of a missed diagnosis. In order to reduce the number of false positives, a proper cut-off value (eg 30\%) may be chosen to obtain high specificity ${ }^{24}$. As with other verification studies, ${ }^{20}$ When $10 \%$ was taken as the cut-off value for malignancy risk, the ADNEX model in the study showed a high negative predictive value; therefore, the model is helpful for excluding malignant tumors.

In this study, acoustic shadows were not present in any malignant tumors but were present in $10.1 \%$ of benign tumors. However, Timmerman et al found that acoustic shadows were present in $17.4 \%$ of benign tumors and $4.2 \%$ of malignant tumors, ${ }^{18}$ which suggests that the number of cases in this study was relatively small, especially the number of cases with borderline, stage I, and metastatic ovarian tumors.

Another limitation of this study is that the diagnostic methods were validated exclusively on patients who underwent surgery, which does not reflect all clinical practice. In addition, the collection of patients was carried out in oncology specialized hospitals, and the prevalence of malignant tumors is high. Although the central type is the weakest influencing factor in the ADNEX model, ${ }^{25}$ it may mean that the results of this study cannot be generalized.

Tug et al found that the diagnostic accuracy of ADNEX model for premenopausal patients with ovarian tumors was higher than that for postmenopausal patients, which was consistent with the results of Sebastian Szubert et al. ${ }^{20,21}$ The original study by the IOTA group did not analyze ADNEX performance according to the menopausal status of the patients. ADNEX does not incorporate the menopausal status of the patients-it focuses only on patient age. ${ }^{6}$ Further studies are needed to determine whether to add menopause to the ADNEX model.

\section{Conclusion}

The ADNEX model shows good diagnostic performance in differentiating between benign and malignant ovarian tumors. The model has a certain clinical value in the 
diagnosis of all subtypes of ovarian tumors, but the sensitivity is poor in the diagnosis of borderline, stage I, and metastatic ovarian tumors and needs to be verified.

\section{Ethics Approval and Consent to Participate}

The study was conducted in accordance with the Declaration of Helsinki (as was revised in 2013). The study was approved by Ethics Committee of the Harbin Medical University Cancer Hospital. The written, informed consent was obtained from all participants. The patient described in Case A provided informed consent for the publication (including all data and images).

\section{Acknowledgments}

We are particularly grateful to all the people who have given us help on our article.

\section{Funding}

This study was funded by the Haiyan scientific research foundation of the Harbin Medical University Cancer Hospital (JJMS2021-02).

\section{Disclosure}

The authors declare that they have no competing interests.

\section{References}

1. Giampaolino P, Della Corte L, Foreste V, et al. Unraveling a difficult diagnosis: the tricks for early recognition of ovarian cancer. Minerva Med. 2019;110(4):279-291. doi:10.23736/S0026-4806.19.06086-5

2. Guo W, Zou X, Xu H, et al. The diagnostic performance of the Gynecologic Imaging Reporting and Data System (GI-RADS) in adnexal masses. Ann Transl Med. 2021;9(5):398. doi:10.21037/atm20-5170

3. Froyman W, Wynants L, Landolfo C, et al. Validation of the performance of International Ovarian Tumor Analysis (IOTA) methods in the diagnosis of early stage ovarian cancer in a non-screening population. Diagnostics (Basel). 2017;7(2):32.

4. Froyman W, Timmerman D. Methods of assessing ovarian masses: international ovarian tumor analysis approach. Obstet Gynecol Clin North Am. 2019;46(4):625-641. doi:10.1016/j.ogc.2019.07.003

5. Sayasneh A, Ferrara L, De Cock B, et al. Evaluating the risk of ovarian cancer before surgery using the ADNEX model: a multicentre external validation study. $B r J$ Cancer. 2016;115 (5):542-548. doi:10.1038/bjc.2016.227

6. Van Calster B, Van Hoorde K, Valentin L, et al. Evaluating the risk of ovarian cancer before surgery using the ADNEX model to differentiate between benign, borderline, early and advanced stage invasive, and secondary metastatic tumours: prospective multicentre diagnostic study. BMJ. 2014;349:g5920. doi:10.1136/bmj.g5920

7. Van Calster B, Van Hoorde K, Froyman W, et al. Practical guidance for applying the ADNEX model from the IOTA group to discriminate between different subtypes of adnexal tumors. Facts Views Vis Obgyn. 2015;7(1):32-41.
8. Timmerman D, Valentin L, Bourne TH, et al. Terms, definitions and measurements to describe the sonographic features of adnexal tumors: a consensus opinion from the International Ovarian Tumor Analysis (IOTA) group. Ultrasound Obstet Gynecol. 2000;16 (5):500-505. doi:10.1046/j.1469-0705.2000.00287.x

9. Prat J. Staging classification for cancer of the ovary, fallopian tube, and peritoneum. Int $J$ Gynaecol Obstet. 2014;124(1):1-5. doi:10.1016/j.ijgo.2013.10.001

10. Meinhold-Heerlein I, Fotopoulou C, Harter P, et al. The new WHO classification of ovarian, fallopian tube, and primary peritoneal cancer and its clinical implications. Arch Gynecol Obstet. 2016;293 (4):695-700. doi:10.1007/s00404-016-4035-8

11. Phinyo P, Patumanond J, Saenrungmuaeng P, et al. Diagnostic added-value of serum CA- 125 on the IOTA simple rules and derivation of practical combined prediction models (IOTA SR X CA-125). Diagnostics (Basel). 2021;11(2):173.

12. Andreotti RF, Timmerman D, Strachowski LM, et al. O-RADS US risk stratification and management system: a consensus guideline from the ACR ovarian-adnexal reporting and data system committee. Radiology. 2020;294(1):168-185. doi:10.1148/ radiol.2019191150

13. Gaurilcikas A, Gedgaudaite M, Cizauskas A, et al. Performance of the IOTA ADNEX model on selected group of patients with borderline ovarian tumours. Medicina (Kaunas). 2020;56(12):690.

14. Nohuz E, De Simone L, Chêne G. Reliability of IOTA score and ADNEX model in the screening of ovarian malignancy in postmenopausal women. J Gynecol Obstet Hum Reprod. 2019;48(2):103-107. doi:10.1016/j.jogoh.2018.04.012

15. Jeong SY, Park BK, Lee YY, Kim TJ. Validation of IOTA-ADNEX model in discriminating characteristics of adnexal masses: a comparison with subjective assessment. J Clin Med. 2020;9 (6):2010. doi: 10.3390/jcm9062010

16. Pereira PN, Sarian LO, Yoshida A, et al. Improving the performance of IOTA simple rules: sonographic assessment of adnexal masses with resource-effective use of a magnetic resonance scoring (ADNEX MR scoring system). Abdom Radiol (NY). 2020;45 (10):3218-3229. doi:10.1007/s00261-019-02207-9

17. Chen H, Qian L, Jiang M, Du Q, Yuan F, Feng W. Performance of IOTA ADNEX model in evaluating adnexal masses in a gynecological oncology center in China. Ultrasound Obstet Gynecol. 2019;54(6):815-822. doi:10.1002/uog.20363

18. Araujo KG, Jales RM, Pereira PN, et al. Performance of the IOTA ADNEX model in preoperative discrimination of adnexal masses in a gynecological oncology center. Ultrasound Obstet Gynecol. 2017;49(6):778-783. doi:10.1002/uog.15963

19. Epstein E, Van Calster B, Timmerman D, Nikman S. Subjective ultrasound assessment, the ADNEX model and ultrasound-guided tru-cut biopsy to differentiate disseminated primary ovarian cancer from metastatic non-ovarian cancer. Ultrasound Obstet Gynecol. 2016;47(1):110-116. doi:10.1002/uog.14892

20. Szubert S, Wojtowicz A, Moszynski R, et al. External validation of the IOTA ADNEX model performed by two independent gynecologic centers. Gynecol Oncol. 2016;142(3):490-495. doi:10.1016/j. ygyno.2016.06.020

21. Tug N, Yassa M, Akif Sargın M, Dogan Taymur B, Sandal K, Mega E. Preoperative discriminating performance of the IOTA-ADNEX model and comparison with Risk of Malignancy Index: an external validation in a non-gynecologic oncology tertiary center. Eur J Gynaecol Oncol. 2020;41(2):20 $0-207$.

22. Joyeux E, Miras T, Masquin I, Duglet PE, Astruc K, Douvier S. Prédictibilité préopératoire de la malignité des tumeurs ovariennes à partir du score ADNEX et utilisation en pratique clinique. Gynecol Obstet Fertil. [Before surgery predictability of malignant ovarian tumors based on ADNEX model and its use in clinical practice]. 2016;44(10):557-564. French. doi:10.1016/j.gyobfe.2016.07.007 
23. Greenhalgh T, Howick J, Maskrey N; Evidence Based Medicine Renaissance Group. Evidence based medicine: a movement in crisis? BMJ 2014;348:g3725. doi:10.1136/bmj.g3725

24. Wynants L, Timmerman D, Verbakel JY, et al. Clinical utility of risk models to refer patients with adnexal masses to specialized oncology care: multicenter external validation using decision curve analysis. Clin Cancer Res. 2017;23(17):5082-5090. doi:10.1158/1078-0432. CCR-16-3248
25. Meys EMJ, Jeelof LS, Achten NMJ, et al. Estimating risk of malignancy in adnexal masses: external validation of the ADNEX model and comparison with other frequently used ultrasound methods. Ultrasound Obstet Gynecol. 2017;49(6):784-792. doi:10.1002/ uog. 17225

\section{Publish your work in this journal}

The International Journal of General Medicine is an international, peer-reviewed open-access journal that focuses on general and internal medicine, pathogenesis, epidemiology, diagnosis, monitoring and treatment protocols. The journal is characterized by the rapid reporting of reviews, original research and clinical studies across all disease areas. The manuscript management system is completely online and includes a very quick and fair peer-review system, which is all easy to use. Visit http://www.dovepress.com/ testimonials.php to read real quotes from published authors.

Submit your manuscript here: https://www.dovepress.com/international-journal-of-general-medicine-journal 